\title{
Observing Exoplanets with the James Webb Space Telescope
}

\author{
Charles A. Beichman \& Thomas P. Greene
}

\begin{abstract}
The census of exoplanets has revealed an enormous variety of planets orbiting stars of all ages and spectral types: planets in orbits of less than a day to frigid worlds in orbits over $100 \mathrm{AU}$; planets with masses 10 times that of Jupiter to planets with masses less than that of Earth; searingly hot planets to temperate planets in the Habitable Zone. The challenge of the coming decade is to move from demography to physical characterization. The James Webb Space Telescope (JWST) is poised to open a revolutionary new phase in our understanding of exoplanets with transit spectroscopy of relatively short period planets and coronagraphic imaging of ones with wide separations from their host stars. This article discusses the wide variety of exoplanet opportunities enabled by JWST's sensitivity and stability, its high angular resolution, and its suite of powerful instruments. These capabilities will advance our understanding of planet formation, brown dwarfs, and the atmospheres of young to mature planets.
\end{abstract}

\section{Introduction}

With a cold $6.5 \mathrm{~m}$ aperture operating in a stable, low-background L2 orbit and equipped with a powerful suite of imaging and spectroscopic instruments, the James Webb Space Telescope (JWST) is poised to revolutionize many areas of astronomy, astrophysics and solar system science when it launches in October 2018 (its current schedule). The study of exoplanets is one of JWST's four primary goals and we shall focus exclusively on this topic in this review. Interested readers are referred to Gardner (2009) for a broader view of JWST's scientific potential.

Charles Beichman

NASA Exoplanet Science Institute, California Institute of Technology and Jet Propulsion Laboratory, 770 S. Wilson St, Pasadena, CA 91105, USA e-mail: chaseipac.caltech.edu

Thomas P. Greene

NASA Ames Research Center, Mountain View, CA e-mail: tom.greene@nasa.gov 
With the initial discovery of 51 Pegasi b in 1995 (Mayor and Queloz 1995) we have gone from knowing of not a single planet beyond our own solar system to a census that now includes more than 3400 confirmed planets discovered using a variety of techniques (Figure 11). As of April 2017, the census includes over 2700 transiting systems of which 2300 come from the Kepler mission, over 600 planets found via the radial velocity technique and 44 planets discovered by direct imaging (http://exoplanetarchive.ipac.caltech.edu/docs/counts_detail.html).

The census work is far from over with on-going and new transit surveys from the ground and from space. These new surveys frequently seek to discover systems that can be characterized further with radial velocity and transit spectroscopy measurements. The TESS and CHEOPS missions, as well as on-going groundbased surveys, promise to identify transiting systems orbiting the brightest and/or very coolest host stars needed for sensitive transit spectroscopic measurements with $H S T, J W S T$, or other high precision observations. On-going coronagraphic surveys using Extreme Adaptive optics on large ground-based telescopes will identify new young, Jupiter-mass planets suitable for characterization via direct imaging and/or spectroscopy with JWST.

Among the surprising demographic results from radial velocity and especially transit surveys are the discovery of hot Jupiters (Mayor and Queloz 1995), a class of mini-Neptunes with radii around $>2-4 \mathbf{R}_{\oplus}$ which have no analog in our own solar system (Dong and Zhu 2013), and the ubiquity of $\sim 1-4 \mathrm{R}_{\oplus}$ planets orbiting cool M stars (Dressing and Charbonneau 2015). As will be discussed below the recent discovery of 7 planets transiting the very cool M8 star, Trappist-1 (Gillon et al. 2017) highlights the opportunities for studying even Earth-sized planets in the coming decade. Each of these classes will provide valuable targets for detailed characterization with JWST.

The exoplanet community has made good first steps in characterizing the physical and chemical properties of a small number of planets with three different techniques: combining transit measurements (which yield the planet's radius) with precision radial velocity (PRV) measurements (which determine its mass) yields the planet's bulk density; measuring the brightness and/or spectrum of a planet during transit, secondary eclipse or through a full orbit yields detailed atmospheric information; making filter photometric or spectroscopic observations of directly imaged planets leading to detailed atmospheric information.

Among the key results from these efforts are: a) the distribution of planet densities, from gas-dominated systems with radii (masses) $>1.5 \mathrm{R}_{\oplus}\left(>5 \mathrm{M}_{\oplus}\right)$ and rocky planets with smaller radii and masses (figure from howard et al 2017); b) the presence of a variety of atomic and molecular species in the atmospheres of transiting and directly imaged planets; $c$ ) the vertical and horizontal thermal structure of exoplanet atmospheres including the presence of supersonic winds and planetary rotation.

But many important questions remain including:

- How do the bulk and atmospheric composition vary as a function of key exoplanet characteristics, such as mass, radius, level of insolation and location within the planetary system? 


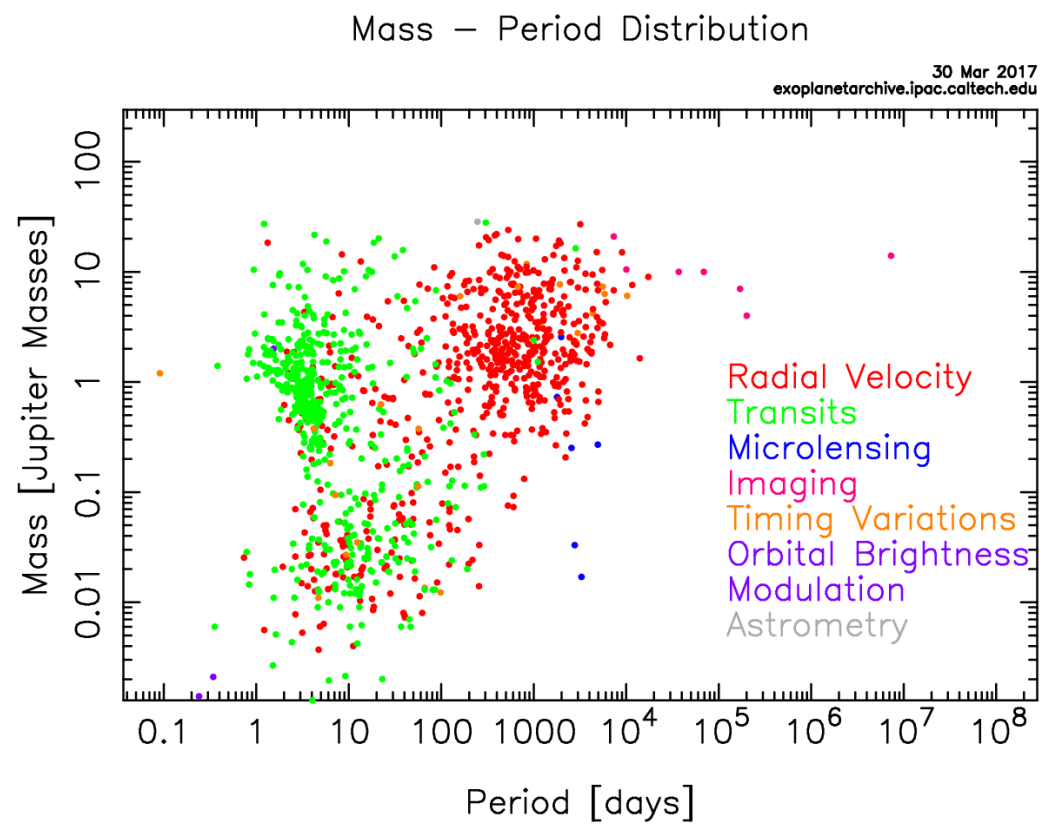

Fig. 1 Census of confirmed exoplanets shows a wide variety of planets in the mass-period parameter space. Different detection techniques are color-coded.

- What can we learn about vertical structure of exoplanet atmospheres and global atmospheric motions?

- What can we learn about the formation of exoplanets from, for example, differences in their atmospheric $\mathrm{C} / \mathrm{O}$ ratio or overall metallicity compared to that of their host star?

- Do massive planets found on distant orbits ( $>50 \mathrm{AU}$ ) have a different formation mechanism compared to those on closer orbits, i.e. disk fragmentation vs. core accretion?

- Can we distinguish between low vs. high initial entropy states for forming massive planets?

- What are the atmospheric constituents of mini-Neptunes, super-Earths, and even terrestrial planets?

- How does the presence of planets affect the structure of debris disks?

- Is the presence of a hot or luminous debris disk a signpost of dynamical activity such as a period of "Late Heavy Bombardment"?

- What can the composition of debris disk material tell us about the formation of planets?

- Do young planetary systems with known massive planets also contain lower mass planets?

JWST is poised to make dramatic advances in all of these areas - and more. In the sections that follow we discuss the various methods of planets searches, and 
more importantly, planet characterization which JWST will bring to the exoplanet community. The ideas described here are drawn primarily from projects being proposed by the instrument teams for the first JWST cycles. The broader community will come up with many new projects, some of these before launch, but the most important and most innovative uses of JWST will doubtless come after launch as we learn about the telescope and the instruments and more importantly as we learn more about exoplanets themselves.

\section{The JWST Instrument Suite}

JWST has four science instruments, and all have modes that will be useful for observing exoplanets:

- NIRCam (Rieke et al. 2005) provides imaging in broad, medium, and narrowband filters as well as grism spectroscopy from 0.6-5 $\mu \mathrm{m}$. In addition, NIRCam has coronagraphic capabilities with a series of Lyot masks, including 3 circular and 2 wedge-shaped occulters (Figure 2, (Krist et al.|2010)).

- NIRspec (Ferruit et al. 2012) provides single,multi-object, and Integral Field (IFS) spectroscopy from $0.7-5 \mu \mathrm{m}$ at spectral resolutions ranging from 100 to 3,000 .

- MIRI (Rieke et al. 2015) provides direct imaging with a variety of filters, slit and IFS spectroscopy at low and medium resolution, and coronagraphy in 4 fixed filters at wavelengths from 5.6 to $28.8 \mu \mathrm{m}$ (Figure 2 (Boccaletti et al. 2015)).

- NIRISS (Doyon et al.2012) provides imaging in a variety of filters out to $5 \mu \mathrm{m}$, wide-field and single-object grism spectroscopy at wavelengths from 0.7-2.5 $\mu \mathrm{m}$, and aperture masking interferometric imaging at 3.8 and $4.8 \mu \mathrm{m}$.

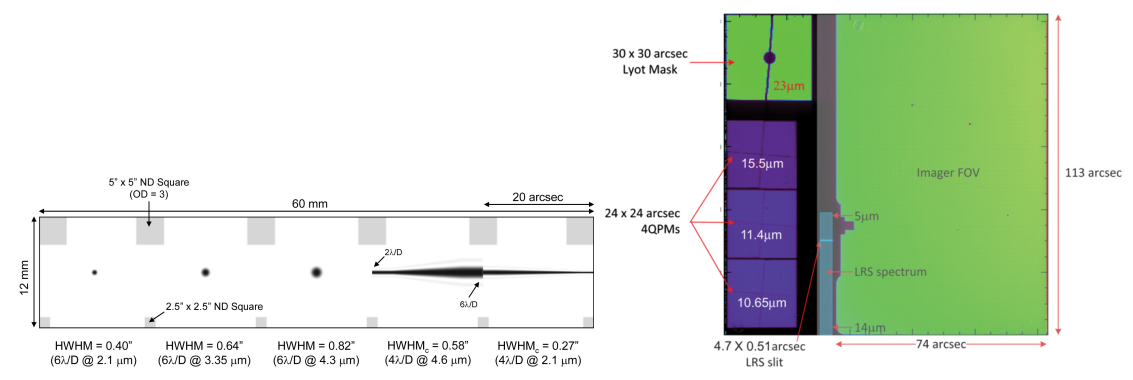

Fig. 2 left) NIRCam has coronagraphic capabilities with a series of Lyot masks, including 3 circular and 2 wedge-shaped occulters optimized for different wavelengths; right) the MIRI focal plane has three Four Quadrant Phase Masks at 10.6,11.4, $15.5 \mu \mathrm{m}$ and a Lyot coronagraph at $23 \mu \mathrm{m}$. 


\section{Coronagraphy and Direct Spectroscopy of Young Planets}

\section{Planet Characterization}

JWST offers unprecedented sensitivity for the characterization of exoplanets via direct imaging and spectroscopy. While JWST does not offer the smallest Wave Front Error (WFE) or the most aggressive Inner Working Angle (IWA) compared with Extreme Adaptive Optics coronagraphs on large ground-based telescopes, its stability, low temperature and low background in space make JWST an extremely sensitive observatory for exoplanet observations longward of $3 \mu \mathrm{m}$ where young planets are bright. JWST is not, however, optimized for carrying out large scale coronagraphic surveys for exoplanets, given the high overheads associated with observations of single targets. Thus, much of JWST's coronagraphic imaging will be focused on characterizing planets within $\sim 2^{\prime \prime}$ of their host stars in previously known systems. NIRSpec's IFS and MIRI's spectrometer will be used to obtain complete spectra of more widely separated planets.

JWST's great strength for direct imaging of planets comes from the stability of its Point Spread Function over a time scale of many hours and its operation in the low background space environment. The Inner Working Angles (IWA) of the NIRCam coronagraphs are relatively coarse, $4 \lambda / \mathrm{D}$ or $6 \lambda / \mathrm{D}$ for the wedge and circular masks, respectively, where D is JWST's $6.5 \mathrm{~m}$ aperture. Thus NIRCam's best IWAs are $0.4^{\prime \prime}$ to $0.6^{\prime \prime}$ at 3.0 and $4.4 \mu \mathrm{m}$. The effective IWA or the MIRI Four Quadrant Phase Masks (FQPM) is roughly $2 \lambda / \mathrm{D}$, or $0.7^{\prime \prime}$ at $11.4 \mu \mathrm{m}$. Contrast limits at $1^{\prime \prime}$ are predicted to approach $10^{-5}$ for NIRCam and $10^{-4}$ for MIRI (Figure 3, Beichman et al. 2010). The on-orbit performance of the coronagraphs will depend sensitively on the drift in telescope wavefront error (WFE) over the duration of a combined target and reference star sequence as well as on the ability of the telescope to hold the star carefully centered on the coronagraphic masks.

The NIRISS Non-Redundant Mask (NRM) or Aperture Masking Interferometer (AMI) operates as an interferometer with an IWA set by the longest baseline across JWST's aperture, roughly $0.5 \lambda / \mathrm{D}$ or $0.07^{\prime \prime}$ at $4.8 \mu \mathrm{m}$, out to an outer working angle defined by the size of an individual aperture in the mask, or about 0.4" (Artigau et al. 2014). The AMI does not block the light of the central star so the sensitivity is affected by the full photon noise of the stellar signal. The AMI mode can operate at 4 wavelengths: $2.8,3.8,4.3$ and $4.8 \mu \mathrm{m}$.

The coronagraphs will be used to characterize previously imaged planets across a suite of NIRCam and MIRI filters. From this information it will be possible to infer such basic properties as total luminosity, effective temperature, and thus effective radius. From this information it will be possible to estimate the initial entropy of the planet which is a direct indicator of its formation mechanism via a "hot" or "cold" start (Figure 5, Marley et al. (2007); Spiegel and Burrows (2012). JWST"s sensitivity is such that it can measure the known young planets at separations of $l e q 1^{\prime \prime}$ in just a few minutes of integration time. Figure 4 shows the signal-to-noise on a 50 MYr old $5 \mathrm{M}_{J u p}$ planet orbiting HR 8799 in a variety of medium and 


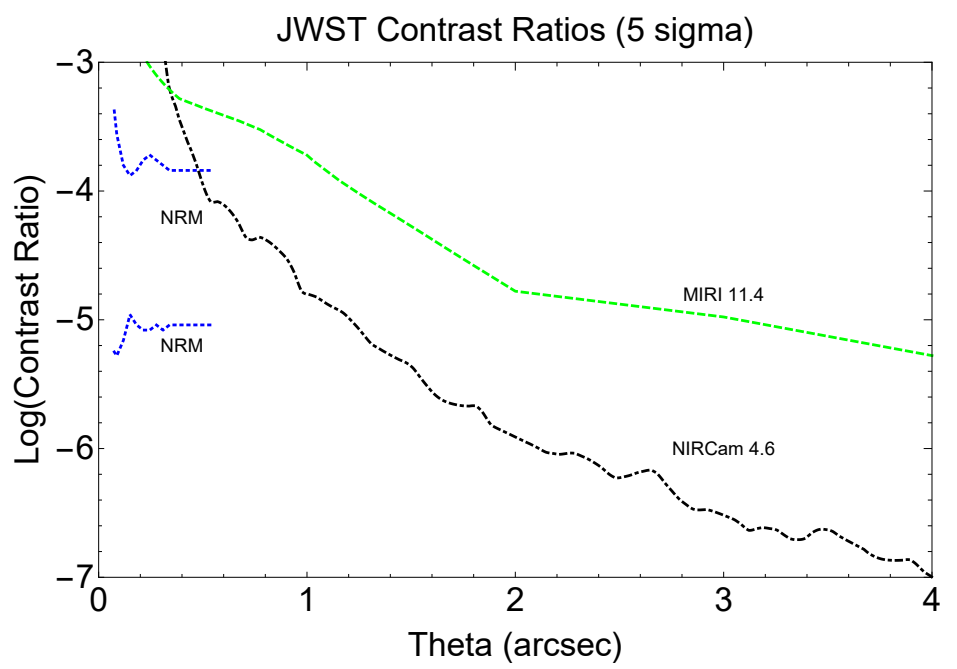

Fig. 3 Contrast ratio cures for JWST's three coronagraphs: MIRI at $11.4 \mu \mathrm{m}$ (green), NIRCam at $4.6 \mu \mathrm{m}$ (black), and the NIRISS Non-redundant Aperture Mask at $4.8 \mu \mathrm{m}$ (the blue dotted lines reflect two assumptions about its sensitivity) Beichman et al. 2010

broad band filters. NIRCam's medium passband filters, F300M, F335M, F360M, F410M, F430M, and F460M will be used to characterize exoplanet atmospheres, measuring both the continuum and searching for signatures of $\mathrm{CH}_{4}, \mathrm{CO}$ and $\mathrm{CO}_{2}$. MIRI's three FQPM filters will isolate a band of $\mathrm{NH}_{3}$ expected in cool $(<1000 \mathrm{~K})$ objects. Illustrative targets for the coronagraphic imaging programs submitted by the instrument teams as given in Table 1 .

Observations using the NIRSpec slit or IFS $(\mathrm{R} \sim 1,000)$ and with the MIRI Low Resolution Spectrometer (LRS, R $\sim 100$ for MIRI) spectrometers will yield high resolution spectra of more widely separated planets. Some of these targets will be challenging due to the proximity of the host star, e.g. the HR8799 planets. Others will be straightforward due to the faintness of the host star, e.g. 2MASS1207-3239, or to the very wide separation between the planet and host, e.g. HD106906 ( $\left.7^{\prime \prime}\right)$, GU Psc $\left(42^{\prime \prime}\right)$ and WD0806-66 (130"). The value of spectroscopy over a broad wavelength range is obvious from examination of Figure 5 and from ground-based observations using the OSIRIS IFS on the Keck telescope (Konopacky et al. 2013) which identified $\mathrm{CO}$ and $\mathrm{H}_{2} \mathrm{O}$ in the atmosphere of HR8799c.

The Aperture Masking Interferometer capability within the NIRISS instrument exploits its very high angular resolution $(0.5 \lambda / D)$ to observe younger, brighter exoplanets located in more distant star forming regions. These include proto-planets, possibly still accreting material, in transition disks such as LkCa15 (Sallum et al. 2015). AMI can also be used to search for few $\mathrm{M}_{J u p}$ planets at 3-5 $\mu \mathrm{m}$ in known systems at separations $<0.25^{\prime \prime}$ with a sensitivity superior to ground-based instruments.

While JWST is not optimized for blind planet searches, there are some programs where JWST offers advantages over ground based facilities. At separations outside 


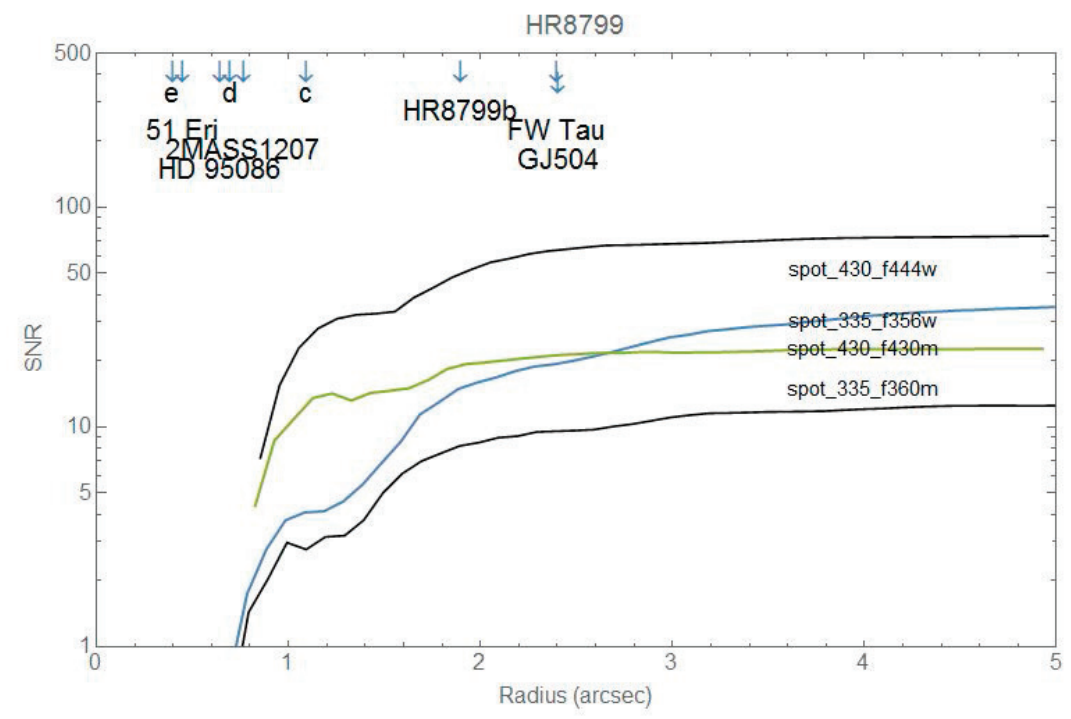

Fig. 4 The signal-to-noise ratio of a $5 \mathrm{M}_{J u p}$ planet orbiting HR8799 in $600 \mathrm{sec}$ is shown as a function of separation from the star at two medium band and two wide band filters using the circular occulters. Planets ' $b$ ' and 'c' are easily detected while planets 'd' and 'e' present more of a challenge. Across the top of the figure are markers showing the angular separations of various well known planets. Close to the star the SNR is dominated by residual stellar photons and speckle subtraction while at larger separations $\left(>2^{\prime \prime}\right)$ the noise for a relatively bright, massive planet is dominated by shot noise from the planet itself.

$1^{\prime \prime} \sim 2^{\prime \prime}$ NIRCam's great sensitivity at 3-5 $\mu \mathrm{m}$ reaches much lower planet masses than is possible from the ground. Hour-long observations in two of NIRCam's wide filters, F322W and F444W, can identify planets with masses below that of Saturn's around previously known planet host stars. Similar searches can be made for $\sim 1$ $\mathrm{M}_{\text {Jup }}$ planets with a 2-10" around 3 of the nearest, brightest debris disk systems. JWST's coronagraphic capabilities will enable a much more sensitive search than was possible with Spitzer (Janson et al.|2015). 
Table 1 Illustrative JWST Targets for Direct Imaging and Spectroscopy

\begin{tabular}{cccccc}
\hline Object & Mass & Age & Separation & & \\
M & Mup $_{\text {I }}$ Myr $)$ & $\left({ }^{\prime \prime}\right)$ & Instrument & Comment $^{a}$ \\
\hline LkCa15 & $0.5 ?$ & 2 & 0.1 & NIRISS & Char,Search \\
HR8 Eri b & 2 & 20 & 0.5 & NIRCam, MIRI & Char, Search \\
HD95086 b & $5-10$ & 50 & $0.6-1.7$ & NIRCam, MIRI, NIRSpec Char, Search, Spectra \\
2MASS 1207-3932 b & 5 & $\sim 15$ & 0.6 & NIRCam, MIRI & Char, Search \\
$\varepsilon$ Eri & $>0.1$ & $\sim 400$ & 0.9 & NIRCam, NIRSpec,MIRI Char, Search, Spectra \\
$\beta$ Leo & $>0.3$ & 45 & $>2$ & NIRCam & Search \\
Vega & $>1$ & 450 & $>2$ & NIRCam & Search \\
2MASS J2236+4751 & $\mathrm{xx}$ & $\mathrm{xx}$ & 3.7 & NIRSpec,MIRI & Search \\
HD106906 b & 11 & 13 & 7.1 & NIRCam, MIRI, NIRSpec & Char, Search \\
Fomalhaut b & $2 ?$ & 15 & 15 & NIRCam, MIRI & Char, Search \\
GU Psc b & 11 & 100 & 42 & NIRSpec,MIRI & Spectra \\
WD 0806-66 & 8 & 2000 & 130 & NIRCam, MIRI, NIRSpec Char, Search, Spectra \\
\hline
\end{tabular}

a "Char" denotes "Characterization" with medium and/or broad band filters; "Search" implies a deep search for planets with masses as low as 1 Saturn-mass;"Spectra" denotes high resolution spectroscopy obtained with either NIRSpec and/or MIRI.

Another promising sample for blind searches is around late $\mathrm{M}$ stars whose hosts are too red or too faint for ground-based Extreme AO instruments. Searches of the youngest and closest $\mathrm{M}$ stars offer the prospect of detecting planets with masses as low as a Uranus mass in orbits as small as 10-20 AU where such planets are thought to form (Schlieder et al. 2015).

\section{Debris Disk and Planets}

JWST will open a dramatic new era in the study of debris disks and their interactions with planets. There is a rich literature of models describing the interaction between planets and disks creating multiple rings, gaps, and various asymmetries (Wyatt 2006, Ertel et al. 2012, Wyatt 2008). The planets responsible for these effects can range in size from Uranus to Jupiter and even down to terrestrial-sized for structures in asteroidal or exo-zodiacal disks (Stark and Kuchner 2008) with $\beta$ Pic (Lagrange et al. 2010) as an example of a warped disk and HD202628 as an example of eccentric, shepherded ring system (Krist et al.2012). NIRCam and MIRI will play complementary roles in advancing our understanding in this area. MIRI's $23 \mu \mathrm{m}$ coronagraph will provide dramatic new images of the closest debris disk systems. Figure 6 shows a sequences of images of the Fomalhaut disk as seen by the Spitzer, HST, and Herschel telescopes along with a simulation of a $23 \mu \mathrm{m}$ image from JWST/MIRI. The offset of the ring from its star has been attributed to the interaction with one or more planets (Acke et al. 2012; Ertel et al. 2012). The spectral energy distribution of Fomalhaut suggests the existence of two rings, a hot $(\sim 170 \mathrm{~K})$ unresolved ring (located around $11 \mathrm{AU}$ or $1.4^{\prime \prime}$ for blackbody grains) and 


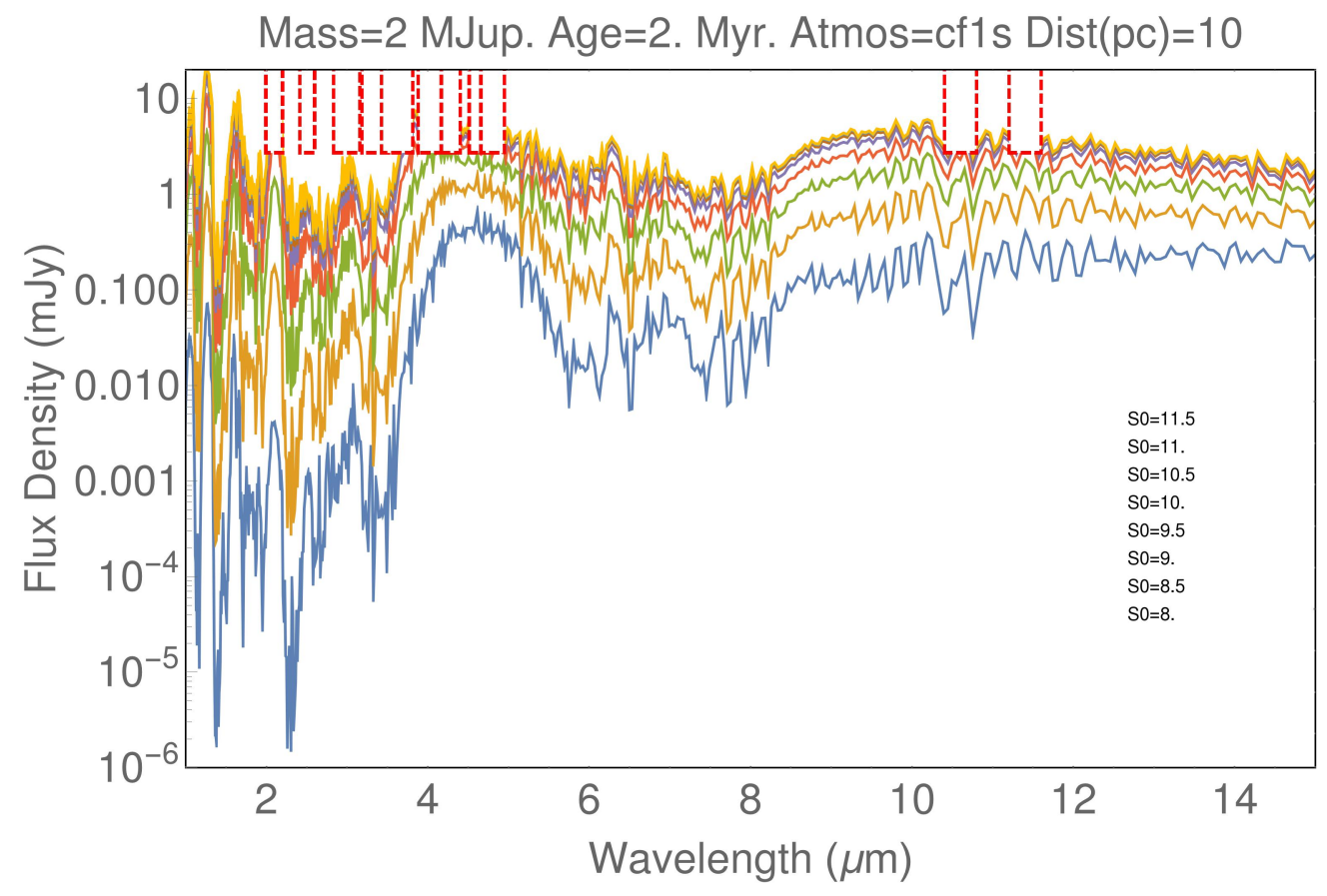

Fig. 5 The emission for a $2 \mathrm{Myr}$ year old $2 \mathrm{M}_{J u p}$ planet for different values of initial entropy (from the brighter "hot" start to fainter "cold" start) based on models by Spiegel and Burrows (2012). The red rectangles across the top of the figure denote the location of selected NIRCam and MIRI filters.

a colder, well resolved one at $\sim 50 \mathrm{~K}$ (Su et al. 2013). With careful PSF subtraction MIRI could resolve both rings with $\sim 1^{\prime \prime}$ resolution, particularly if the grains in the inner ring are small $(\lambda<<23 \mu \mathrm{m})$ and thus located beyond $11 \mathrm{AU}$. Fomalhaut has already been identified as having one planet in visible images, Fomalhaut $b$ (Kalas et al.|2008), although the lack of detectable near-IR emission and the highly eccentric, ring-crossing orbit of the "planet" have given rise to the idea that the planet may be a dust cloud created by a collision of two comets (Lawler et al.2015).

Complementing MIRI's disk images will be imaging with the NIRCam coronagraph to search for 3 and $4 \mu \mathrm{m}$ emission from Fomalhaut $\mathrm{b}$ in addition to any other planets which might be present. NIRCam can detect or set limits on the presence of planets at separations of $>1-2^{\prime \prime}$ for the closest, youngest debris disk systems: with mass limits as small as Jupiter's (Vega, Fomalhaut, $\beta$ Leo) or Saturn's for $\varepsilon$ Eri. Unfortunately, for more distant or older systems ( $>1 \mathrm{Gyr}$ ) all but the most massive planets (or brown dwarfs) will be either too cold or too close to their host star to be detected with NIRCam. 

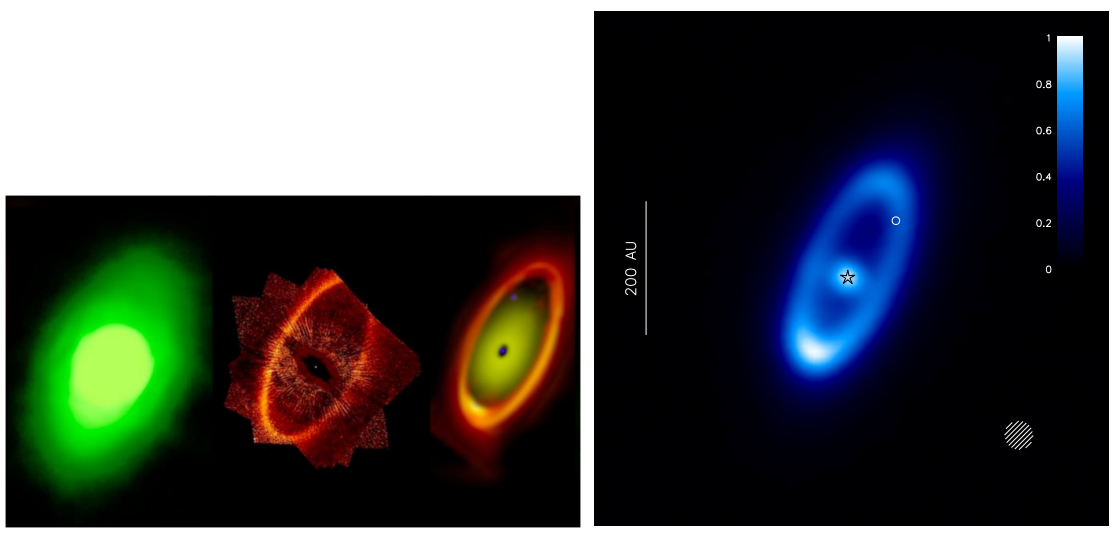

Fig. 6 left) A comparison of the Spitzer $24 \mu \mathrm{m}$ and HST visible light images of the Fomalhaut disk with a simulation of a MIRI $23 \mu \mathrm{m}$ image (courtesy George Rieke). The disk seen at visible wavelengths is fully resolved with $\sim 1^{\prime \prime}$ resolution in the MIRI image. The brighter ansa is due to the ring being offset from the star and the closer grains being heated more strongly. right) A 70 $\mu \mathrm{m}$ image with 5.7" resolution of the Fomalhaut disk obtained with the Herschel telescope (Acke et al. 2012).

JWST will explore the morphology and composition of disks via filter photometry with the NIRCam coronagraph looking for evidence of ices and tholins which characterize Kuiper Belt objects in our own solar system. The MIRI and NIRSpec spectrometers will explore spatially unresolved disks including many of the disk systems with $22 \mu \mathrm{m}$ emission discovered by the WISE satellite (Patel et al.|2014). The compositional information may yield surprises such as the silica grains found toward $\eta$ Corvi which were interpreted to be due the remnants of a very energetic collisional event, perhaps the impact of a large Kuiper Belt object onto a terrestrialsized planet (Lisse et al. 2012; $\mathrm{Su}$ 2015).

\section{Brown Dwarfs}

Brown Dwarfs (BDs) are the more massive cousins of Jovian-mass planets, starting at the Deuterium burning limit of $13 \mathrm{M}_{J u p}$ and extending upward to the Hydrogen burning limit of $70 \mathrm{M}_{\text {Jup }}$. The first examples, the L dwarfs with effective temperatures $\leq 2000 \mathrm{~K}$ were discovered in Sloan (SDSS) and 2MASS sky surveys of the 1990s (Kirkpatrick et al. 1999) and as bound companions to nearby stars, e.g GJ1229B (Nakajima et al. 1995). Subsequently, the WISE survey pushed the detection limits to cooler temperatures, the $\mathrm{T}$ and $\mathrm{Y}$ classes with temperatures below $1000 \mathrm{~K}$ (Kirkpatrick 2013).

The space motions of BDs are similar to those of late type M dwarfs (Faherty et al. 2012), so it is likely that these objects represent the lowest mass end of the 
initial mass function, slowing falling in number as the efficiency of star formation decreases to lower mass (Kirkpatrick et al.2011).

Because the temperature and luminosity of BDs slowly decline with age (Burrows et al. 1989) determining the mass of a BDs of unknown age is a challenge. Some BDs, found in young clusters, are quite hot, i.e. appearing as $\mathrm{L}$ dwarf or even a late $\mathrm{M}$ dwarf, but not very massive, such as $8 \mathrm{M}_{J u p}$ identified in Chamaeleon (Luhman et al. 2005). These young objects can be identified through their association with clusters and through spectroscopic features such as low surface gravity (Gagné et al.2014). JWST imaging surveys with NIRCam of young clusters could identify many more such objects.

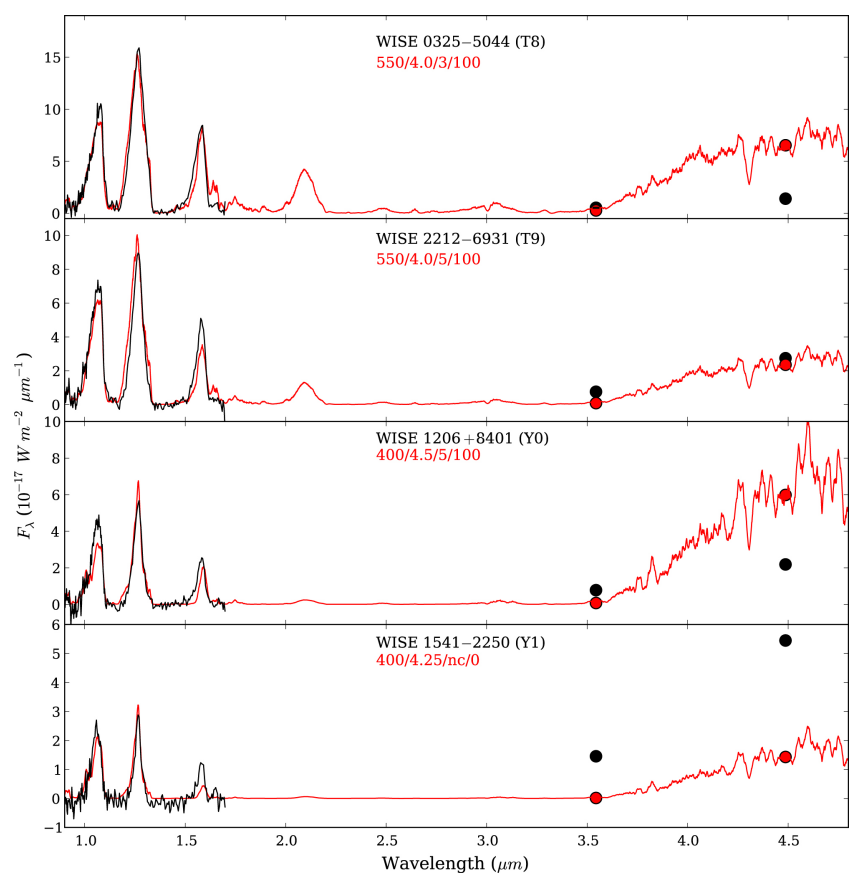

Fig. 7 The spectra of 4 late $\mathrm{T}$ and $\mathrm{Y}$ dwarfs from 1 to $5 \mu \mathrm{m}$ showing fits to various models. Models fitted to the short wavelength data provide a progressively poorer fits (red circles) to the photometric data at longer wavelengths (black circles) for the cooler spectral types (Schneider et al. 2015).

Among the most dramatic discoveries of the WISE mission was the detection of the coldest $(<400 \mathrm{~K})$, lowest mass $\left(\sim 5-10 \mathrm{M}_{\text {Jup }}\right)$ Y dwarfs located close to the sun. WISE 0855-0714 is a high proper motion BD located just $2.2 \mathrm{pc}$ away. Its effective temperature is just $250 \mathrm{~K}$ and its inferred mass just $5 \mathrm{M}_{\text {Jup }}$ assuming a typical field age of 2-10 Gyr (Luhman 2014). WISE $1828+2650$ is a similarly cold Y dwarf located only $9.4 \mathrm{pc}$ away (Beichman et al. 2014b). Spitzer identified a bound companion to the nearby white dwarf WD0806-661 located just $19.2 \mathrm{pc}$ away and 
orbiting its host in a $2500 \mathrm{AU}$ orbit has an 300-345 K effecitve temperature and an inferred mass of 6-9 $\mathrm{M}_{J u p}$ (Luhman et al. 2012).

Although the free-floating and bound but widely separated BDs may have a different formation histories from one another and from planets formed via core accretion, these objects will serve as a critical laboratories for models of exoplanet and $\mathrm{BD}$ atmospheres. Because they are free from the glare of a host star, it is straightforward to bring the full power of JWST's NIRSpec and MIRI spectrometers to bear on their study. These investigations will be particularly important since the spectra and overall spectral energy distributions for $\mathrm{Y}$ dwarfs are a very poor fit to the observations, failing in some cases by an order of magnitude or more for the lowest temperature objects (Figure 7, Beichman et al. (2014b); Schneider et al. (2015)).

Important JWST programs will include complete 3-15 $\mu \mathrm{m}$ spectra at $\mathrm{R} \sim 1,000-$ 3,000 across the whole range of $\mathrm{L}, \mathrm{T}$ and $\mathrm{Y}$ dwarfs with an emphasis on the coldest, lowest mass objects which are close analogs to mature exoplanets. Measurement of the full spectral energy distribution combined with known distances to selected objects can be used to assess the bolometric luminosity. This information, combined with an assumption of radius $\sim 1 \mathrm{R}_{\text {Jup }}$ for a mature $\mathrm{BD}$, will constrain effective temperature and thus the atmospheric models. Comparison of various molecular species seen in $\mathrm{T}$ and $\mathrm{Y}$ dwarfs with those seen in transiting exoplanets may reveal differences in abundances which might be traced to their respective formation histories, i.e. evidence for core accretion in an increased metallicity relative to standard stellar abundances.

Other valuable targets will include binary BD-BD systems for which it will be possible to determine individual $\mathrm{BD}$ masses and companion star-BD systems such as HD 19467 which hosts a $50 \mathrm{M}_{\text {Jup }}$ T5.5 brown dwarf but whose age and initial metallicity are known from the properties of the host star and its mass from radial velocity measurements (Crepp et al. 2015).

Finally, there is evidence of rapid rotation in brown dwarfs as well "weather", i.e. flux and color variability due to the formation of clouds or other phenomena (Biller et al. 2015). Spectroscopy study of these systems using NIRSpec and MIRI will be important JWST programs.

\section{Transiting Planets: Transmission and Emission Spectroscopy}

We now discuss how JWST will likely advance the characterization of the atmospheres of transiting planets. The JWST primary mirror area is just over $25 \mathrm{~m}^{2}$, about 5.6 times that of $H S T$ and almost exactly 50 times the collecting area of Spitzer. This means that $R \simeq 200$ JWST spectra will have comparable $\mathrm{S} / \mathrm{N}$ to $H S T$ $R \simeq 35$ near-IR spectra and Spitzer IRAC $R \simeq 4.5$ mid-IR photometric observations of equal length. Thus JWST will produce considerably higher resolution spectra than obtained by HST and will also provide the first high S/N mid-IR emission and transmission spectra of exoplanet atmospheres. 


\section{JWST Transit Science Opportunities}

JWST will have sufficient sensitivity and spectral range to make high SNR spectral observations of transiting exoplanets that span numerous molecular features over wavelengths from less than 1 to more than 10 microns. Transmission spectra taken during and adjacent to transits reveal the compositions of high atmospheres at low pressures $(P \sim 1$ mbar) but are often impacted by high altitude hazes, aerosols, or clouds. Emission spectra taken during and adjacent to secondary eclipses reveal planets temperature-pressure profiles and compositions at higher pressures, sampling a greater region of their atmospheres. Feature strengths of the common $\mathrm{CH}_{4}$, $\mathrm{CO}, \mathrm{CO}_{2}, \mathrm{H}_{2} \mathrm{O}$, and $\mathrm{NH}_{3}$ (not yet detected) molecules infer a planet's $\mathrm{C} / \mathrm{O}$ ratio and overall metal abundance, constraining its formation history. JWST will be able to obtain high quality transmission and emission spectra of numerous warm-to-hot planets down to $\sim 10$ Earth masses or less (Greene et al. 2016, Rocchetto et al. 2016; Mollière et al. 2017), advancing exoplanet characterization into a new era.

Hot Jupiters are known to have strong atomic $\mathrm{Na}$ and $\mathrm{K}$ absorption features and Rayleigh slopes in red visible wavelengths (e.g., see Crossfield 2015, Sing et al. 2016). Strong $\mathrm{H}_{2} \mathrm{O}$ features have also been detected in the 1.1 - 1.7 $\mu \mathrm{m}$ HST WFC3 transmission or emission spectra of several planets, important for establishing atmospheric metallicity. Figure 8 shows these $\mathrm{H}_{2} \mathrm{O}$ absorptions as well as longer wavelength $1.7-11 \mu \mathrm{m}$ features of $\mathrm{CH}_{4}, \mathrm{CO}, \mathrm{CO}_{2}$, and $\mathrm{NH}_{3}$ that vary with temperature and will be diagnostic of the atmospheric composition and chemistry of cool to hot $\left(400 \mathrm{~K} \leq T_{\mathrm{eq}} \leq 3000 \mathrm{~K}\right.$ planetary atmospheres.

\section{Optimal JWST Instrument Modes}

The four JWST science instruments all have modes that will be useful for observations of transiting planets (e.g., see Beichman et al. 2014a; Batalha and Line 2017, Howe et al. 2017). Modes with high throughput, high time efficiency, good brightness limits, fine spatial sampling (producing well-resolved PSFs), and slitless or wide-slit spectroscopic operation will provide the highest possible precision, necessary for detecting atmospheric features in exoplanets in the presences of their extremely bright host stars. Using these qualifications, we present the best suited for time series spectra of transiting planets in Table 2 and discuss them below. The imaging modes of NIRCam and MIRI are also well-suited to exoplanet transit and secondary eclipse observations, but we do not discuss them further. All of the modes mentioned here will be available for acquiring data in time series exposures without dithering, and more information on the JWST science instruments is available at https://jwst-docs.stsci.edu/display/JTI

NIRISS SOSS mode uses a unique grism to produce a slitless spectrum spanning $0.6-2.8 \mu \mathrm{m}$ in 3 orders simultaneously. This provides $R \equiv \lambda / \delta \lambda \simeq 700$ resolving power, and a powered surface on the grism spreads the a point source over $\sim 25$ 


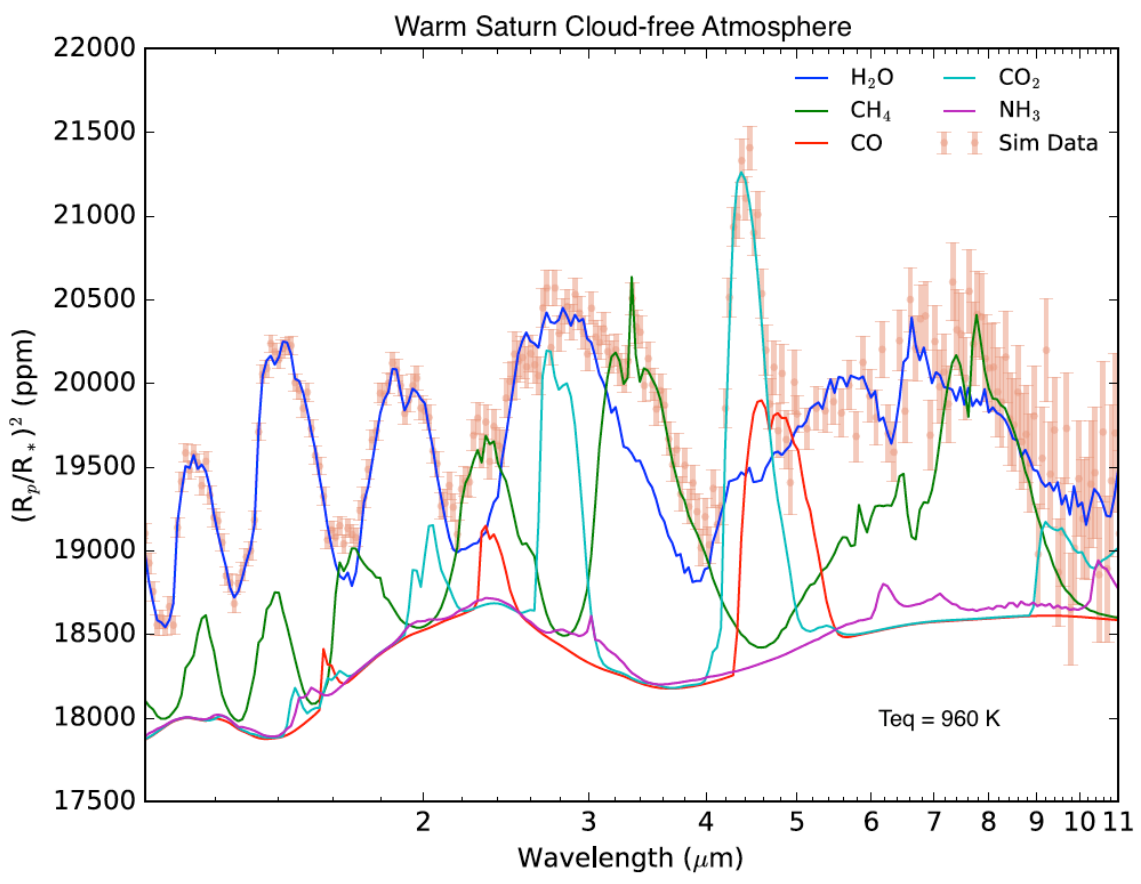

Fig. 8 Equilibrium molecular feature strengths and simulated $J W S T$ spectrum of a warm $\left(T_{\mathrm{eq}}=\right.$ $960 \mathrm{~K}$ ) sub-Saturn mass planet with a H-dominated, cloud-free atmosphere (similar parameters to (similar to HAT-P-12 b). Model spectra were created using the CHIMERA suite (Line et al. 2013 2014), and simulated JWST observations for 1 transit at each wavelength are also shown. Observations of planets in this temperature range will probe the transition from $\mathrm{CO}+\mathrm{CO}_{2}$ to $\mathrm{CH}_{4}$ dominance in exoplanet atmospheres and will sensitively detect non-equilibrium chemistry. Figure was provided by E. Schlawin.

Table 2 JWST modes best suited for transiting planet spectra

\begin{tabular}{lllllll}
\hline Instrument & Mode & $\begin{array}{l}\lambda \\
(\mu \mathrm{m})\end{array}$ & $\begin{array}{l}R \\
\lambda / \delta \lambda\end{array}$ & $\begin{array}{l}\text { PSF } \\
\text { (pixels) }\end{array}$ & $\begin{array}{l}\text { Saturation } \\
(\mathrm{K} \text { mag })\end{array}$ & Comment \\
\hline NIRISS & SOSS & $0.6-2.8$ & $\sim 700$ & $\sim 25$ & $6.2-7.5$ & Slitless \\
NIRSpec & Prism & $0.6-5.3$ & $\sim 100$ & $<2$ & 10.2 & Wide Slit BOTS \\
NIRSpec & G140M/H+F100LP & $1.0-1.9$ & $\sim 1000 / 2700$ & $<2$ & $8.0 / 6.8$ & Wide Slit BOTS \\
NIRSpec & G235M/H+F170LP & $1.7-3.2$ & $\sim 1000 / 2700$ & $<2$ & $7.5 / 6.3$ & Wide Slit BOTS \\
NIRSpec & G395M/H+F290LP & $2.9-5.3$ & $\sim 1000 / 2700$ & $<2$ & $6.5 / 5.5$ & Wide Slit BOTS \\
NIRCam & Grism+F322W2 & $2.4-4.0$ & $\sim 1500$ & $\leq 2$ & 4.4 & Slitless \\
NIRCam & Grism+F444W & $3.9-5.0$ & $\sim 1500$ & $\geq 2$ & 3.7 & Slitless \\
MIRI & LRS & $\sim 5-\sim 12$ & $\sim 100$ & $<2-3$ & 5.7 & SLITLESSPRISM \\
\hline
\end{tabular}


pixels in the spatial direction to improve bright limits and maintain high precision in the presence of any detector intrapixel response variations telescope jitter (e.g., see Deming et al. 2009: Beichman et al.|2014a). The three orders are spatially separated at most wavelengths.

NIRCam has $\mathrm{Si}$ grisms that produce $R=1100-1700$ slitless spectra over 2.4 $5.0 \mu \mathrm{m}$ wavelengths (Greene et al. 2017 JATIS submitted). The high dispersion of the grism and limited size of the detector requires observations in at least 2 filters to cover this full spectral range. The dichroic design of NIRCam allows simultaneous shorter wavelength observations of the same field, and several medium and narrowband filters with weak lenses to spread the image over many pixels will be available in Cycle 1 JWST observations. We expect that the NIRCam dispersed Hartmann sensor (DHS) will be available for scientific use starting in Cycle 2 (observations taken in April 2020 and later). This will allow simultaneous $R \simeq 3001-2 \mu \mathrm{m}$ and a subset of $2.4-5.0 \mu \mathrm{m}$ spectroscopy (Schlawin et al. 2017).

MIRI LRS provides a slitless mode that acquires low resolution $(R \sim 100)$ spectra over $5-\sim 12 \mu \mathrm{m}$ wavelengths (Kendrew et al. 2015). Like all slitless spectrographs, each MIRI LRS pixel sees nearly the full background of that bandpass, which amounts to $\sim 200-300$ electrons s ${ }^{-1}$ pixel $^{-1}$. Transmission losses, increased dispersion, increased diffraction, and this background reduces the $\mathrm{S} / \mathrm{N}$ at longer wavelengths, so this mode will be most useful at $\lambda \leq 10 \mu \mathrm{m}$.

NIRSpec provides a wide, $1.6^{\prime \prime} \times 1.6^{\prime \prime}$ slit that is intended to minimize slit modulation of spectra in the presence of telescope jitter (Ferruit et al. 2014). All of the 6 NIRSpec gratings and prism (Table 2) can be used for time series transit spectroscopy with this aperture. Table 2 also shows NIRSpec provides wider simultaneous $3--5 \mu$ spectroscopy than NIRCam, but NIRCam has finer spatial sampling (64 vs. 100 mas pixel $^{-1}$ ), and and is truly slitless. NIRSpec covers more spectral range in a given exposure, but NIRCam may provide closer to photon-limited precision on very bright stars. This will not be known for sure until after JWST in-orbit commissioning.

Spectrophotometric precision of JWST data will be impacted by astrophysical photon noise, astrophysical systematic noise (e.g., starspots), slit effects and detector intrapixel response variations in the presence of telescope jitter, detector stability, and perhaps background stability for the MIRI LRS. The $1-5 \mu \mathrm{m} \mathrm{HgCdTe}$ detectors in NIRISS, NIRCam, and NIRSpec have similar architectures to that in HST WFC3 which has achieved excellent stability on the order of $20 \mathrm{ppm}$. However, the $J W S T$ have different readout control electronics and signal amplifiers, provided by a single SIDECAR ASIC per detector. These ASICS may or may not prove to be less stable than the more discrete HST WFC3 electronics, but we will not know until JWST is commissioned on orbit. MIRI uses Si:As detectors, control, and readout electronics that have Spitzer IRAC heritage, so we expect MIRI LRS and imager precision to be similar to that of the $\lambda>5 \mu \mathrm{m}$ channels of SPitzer IRAC. 


\section{Observations of Archetypal Transiting Systems}

Several studies have recently simulated JWST spectra of a variety of planet types and have assessed what can be learned from these data from direct examination of spectra (Deming et al. 2009; Barstow and Irwin 2016; Mollière et al. 2017) or statistical Bayesian retrieval techniques (Barstow et al. 2015: Greene et al. 2016, Rocchetto et al.2016; Batalha and Line 2017; Howe et al. 2017). We now draw from these works to discuss the expected data quality and molecular features that may be detectable in JWST spectra of several different types of exoplanet atmospheres. The example spectra shown in this subsection are produced from the simulations by Greene et al. (2016), but Mollière et al. (2017) and the other works cited above present numerous other examples.

Hot Jupiters with bright host stars will generally have high $\mathrm{S} / \mathrm{N}$ transmission and emission spectra in a single transit or secondary eclipse, respectively. Figure 9 shows that a hot Jupiter like HD 209458 b would have absorption features of several hundred parts per million (ppm) if its atmosphere was cloud-free, and these would be detected at high $\mathrm{S} / \mathrm{N}$. Even a cloudy atmosphere (opaque cloud at 1 mbar) would show $\sim 100$ ppm features of $\mathrm{H}_{2} \mathrm{O}, \mathrm{CO}$, and $\mathrm{CO}_{2}$ that would be detected at high confidence.

Warm Neptune planets with masses $M \sim 20 M_{\oplus}$ and equilibrium temperatures 600 $\mathrm{K}<T_{\text {eq }}<1000 \mathrm{~K}$ will also be amenable to characterization with JWST transmission and emission spectroscopy. Transmission feature strengths can also be several hundred ppm in size for $\mathrm{M}$ dwarf host stars, and strong $\mathrm{H}_{2} \mathrm{O}$ and $\mathrm{CH}_{4}$ features can be detected at high $\mathrm{S} / \mathrm{N}$ for bright host stars, $K \leq \sim 7$ mag. Figure 10 shows that a $T_{\text {eq }}=700 \mathrm{~K}$ planet like GJ $436 \mathrm{~b}$ will a have detectable emission spectra at $\lambda>4$ $\mu \mathrm{m}$. H-dominated mini-Neptune planets $\left(R \simeq 2 R_{\odot}\right)$ with bright $\mathrm{M}$ star hosts are also expected to have similarly strong transmission and emission features.

Super-Earth and Earth atmospheres will be difficult to characterize with JWST; numerous transmission or emission spectra will likely need to be co-added to make confident detections of their weak features. Schwieterman et al. (2016) have shown that even the strongest near-IR spectral features of an Earth-like planet with an $\mathrm{M}$ dwarf host will have amplitudes of under $\sim 10 \mathrm{ppm}$ in transmission. Barstow et al. (2016) find that Earth and Venus atmospheres have some mid-IR features $\left(\mathrm{CO}_{2}, \mathrm{O}_{3}\right)$ that are somewhat stronger but still well under $100 \mathrm{ppm}$. The warmer TRAPPIST-1 planets (Gillon et al.2017) will likely have continuum emission on the order of 100 ppm or more compared to their host star at $\lambda>10 \mu \mathrm{m}$, and it may be possible to detect strong $\mathrm{CO}_{2}$ or other mid-IR features at these wavelengths in several or many MIRI photometric observations (see Deming et al. 2009).

Proxima b, the nearest planet, is not currently known to transit. However, Kreidberg and Loeb (2016) predict that its day / night contrast should produce a $\sim 30-100 \mathrm{ppm}$ signal at $\lambda \geq 15 \mu \mathrm{m}$ if its atmosphere redistributes $35 \%$ to $0 \%$ of its absorbed incident flux. It is possible that the JWST MIRI instrument may de- 


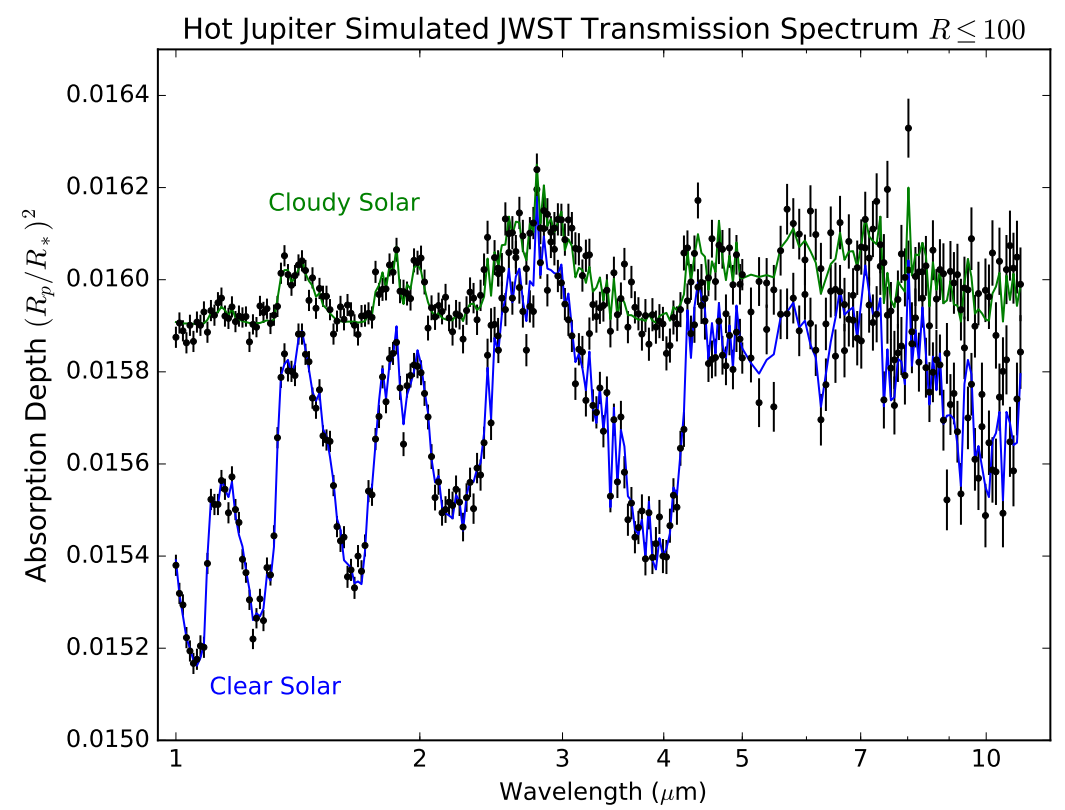

Fig. 9 Clear (blue curve) and cloudy (green curve) chemical equilibrium models of a hot Jupiter planet with solar composition. Simulated JWST NIRISS SOSS, NIRCam grism, and MIRI LRS data are shown as black points and error bars. The planet and stellar parameters of the HD 209458 $\mathrm{b}$ system were used, and the observations were binned to $R \leq 100$. One transit and an equal time on the star mush be observed in each instrument mode (4 total) to cover this spectral range. See Greene et al. (2016) for more details.

tect this signal in one or more phase curve observations, but the 11 day orbit of the planet will make this an exceptionally challenging measurement.

\section{Expected Characterization Results}

Figures Figure $8-10$ and the studies cited above show that JWST spectra will show numerous molecular features that will be useful for characterizing large-to-small and hot-to-warm planets. We now examine how well major atmospheric parameters can be constrained by JWST spectra of transiting planet systems.

Atmospheric compositions can be constrained well with transmission spectra of warm Neptunes to hot Jupiters that have clear atmospheres with solar to $\sim 100 \times$ solar metallicities $(0 \leq[\mathrm{M} / \mathrm{H}] \leq 2)$. Using information retrieval techniques, Greene et al. (2016) show that $\mathrm{H}_{2} \mathrm{O}$ and $\mathrm{CO}$ (the dominant molecular components) can be 


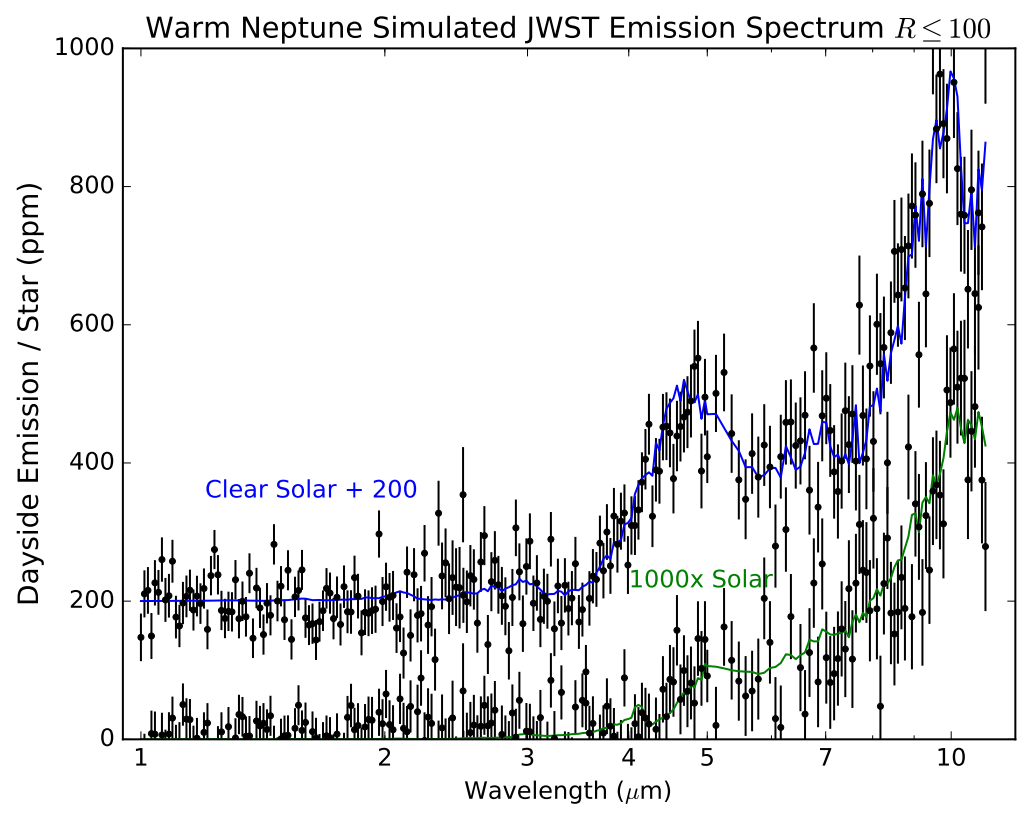

Fig. 10 Solar abundance (blue curve) and high mean molecular weight $(1000 \times$ solar, green curve) emission spectra of a warm Neptune planet in chemical equilibrium. Simulated JWST NIRISS SOSS, NIRCam grism, and MIRI LRS data are shown as black points with error bars. The planet and stellar parameters of the GJ $436 \mathrm{~b}$ system were used, and the observations were binned to $R \leq 100$. One secondary eclipse and an equal time on the star+planet dayside must be observed in each instrument mode (4 total) to cover this spectral range. See Greene et al. (2016) for more details.

constrained to factors of 1.5 - 2 with transmission spectra of hot Jupiter planets in favorable systems. The mixing ratios of $\mathrm{H}_{2} \mathrm{O}$ and $\mathrm{CH}_{4}$, the dominant molecules in cooler atmospheres, can be constrained to a factor of 3-10 for warm Neptune and sub-Neptune planets with clear atmospheres. These results are for a single transit at each wavelength. Complete $<1-11 \mu \mathrm{m}$ spectra provide the tighter constraints in these ranges, while NIRISS SOSS $(\lambda \leq 2.5 \mu \mathrm{m})$ alone provides the looser ones. Emission spectra of these systems provide similar constraints for Hot Jupiters if the entire $<1-11 \mu \mathrm{m}$ spectrum is observed, but this degrades to a factor of $\sim 4$ if only NIRISS SOSS spectra are acquired. Emission spectra of warm Neptunes and sub-Neptunes can constrain $\mathrm{H}_{2} \mathrm{O}$ and $\mathrm{CH}_{4}$ mixing ratios to only a factor of $\sim 10$.

C/O ratios and metallicity provide clues to a planet's formation history. Öberg et al. (2011) show that the $\mathrm{C} / \mathrm{O}$ ratio of the gas in a protoplanetary disk increases beyond its $\mathrm{H}_{2} \mathrm{O}$ and $\mathrm{CO}_{2}$ ice lines, so planets formed beyond these locations may be expected to have $\mathrm{C} / \mathrm{O}$ ratios above solar $(0.55)$ or their parent star if there is little mixing in the disk. Mordasini et al. (2016) develop this concept further by addi- 
tionally considering how accretion of planetesimals, migration, and thermodynamic evolution impact the chemistry of hot Jupiters and they model resultant transmission and emission spectra. They find that a number of pathways to achieve solar-like, oxygen-rich $\mathrm{C} / \mathrm{O}<1$ values; hot Jupiters with $\mathrm{C} / \mathrm{O}>1$ are possible but unlikely. In addition to $\mathrm{C} / \mathrm{O}$, overall planet metal enrichment $[\mathrm{M} / \mathrm{H}]$ may also be related to formation scenarios. Gas and ice giant solar system planets having $[\mathrm{M} / \mathrm{H}]$ enhancements increase by a factor of $\sim 30$ as their masses decrease from Jupiter to Uranus (Kreidberg et al. 2014).

These formation scenarios can be investigated by measuring the $\mathrm{C} / \mathrm{O}$ ratios of exoplanet atmospheres with JWST. Greene et al. (2016) show that JWST spectra will likely be able to constrain $\mathrm{C} / \mathrm{O}$ ratios to within $\sim 50 \%$ for transmission or emission spectra of clear or cloudy hot Jupiters optained over a single transit or secondary eclipse at all wavelenghts $1-11 \mu \mathrm{m}$. Warm Neptunes and sub-Neptunes are somewhat worse, with typical $1 \sigma \mathrm{C} / \mathrm{O}$ constraints from $\sim 50 \%$ to a factor of $\sim 3$ in most transmission scenarios. They also find that full-wavelength $J W S T$ spectra constrain $[\mathrm{M} / \mathrm{H}]$ values to about a factor of 3 in these planets, so the enrichment seen in the solar system should be detected in JWST spectra with high confidence.

3-D thermal profiles, chemical disequilibrium, and cloud properties will also be revealed in JWST spectra. Emission spectra will reveal dayside temperaturepressure profiles over a large pressure range $\sim 1$ bar (e.g. Line et al. 2014), and transmission spectra will measure the temperatures and compositions in planet terminators. Numerous planets will be amenable to both transmission and emission spectroscopy (e.g., Greene et al. 2016; Mordasini et al. 2016), enabling 3-D mapping of temperatures and compositions. Phase curve spectra will also reveal global circulation patterns in these parameters. Chemical disequilibrium due to vertical mixing or photochemistry (e.g., Line and Yung 2013, Shabram et al. 2011) should also be detectable in JWST spectra of transiting planets. Finally, cloud particles will contribute to the mid-IR emission spectra of hot planets (e.g., Crossfield 2015), and cloud-top pressures can be retrieved from transmission spectra (Greene et al.2016).

\section{Improved Information Retrieval}

Modern information retrieval techniques for transiting exoplanet spectra (Line et al. 2013, 2014, Benneke 2015) have generally been developed and refined using modest resolution data typically spanning under an octave (e.g., HST WFC3 G141 1.1 - $1.7 \mu \mathrm{m})$. JWST spectra will have higher spectral resolution over much larger spectral range (see Table 2. Rocchetto et al. (2016) find that the large $1-10 \mu \mathrm{m}$ bandwidth of $J W S T$ spectra will require improving retrievals to include allowing temperature variation with altitude in transmission spectra. This may seem unusual given that transmission spectra are generally thought to be sensitive to absorbers only at the highest altitudes. However, the particular altitude probed is a fairly strong function of wavelength, so retrieving atmospheric parameters over a large 
wavelength range will sample a range of temperatures. Real JWST spectra will doubtlessly present more retrieval challenges, forcing improvements in chemical networks, temperature-pressure profile parameters, cloud models, and likely other factors as well.

\section{Expecting the Unexpected}

An observatory as powerful as JWST will undoubtedly produce many surprises. Just as Spitzer was never designed to observe planets but has proven to be a critical facility for observing transiting exoplanets, so too may JWST surprise us with its capabilities. We consider two programs which will stretch the instruments and the telescope, and the tolerance of the Time Allocation Committees, but which might lead to the discovery and characterization of one or more nearby Earths.

\section{Detecting an Earth Around the Nearest Stars}

The detection and characterization of an Earth-sized planet in the Habitable Zone of a nearby solar type star represents the culmination of one of NASA's strategic goals. A variety of NASA mission concepts, from Darwin (Fridlund 2004) and the Terrestrial Planet Finder (Beichman et al. 2007) to concepts presently under study including a starshade for WFIRST (Seager et al. 2015) and the LUVOIR (DomagalGoldman et al. 2017) and HabEx (Mennesson et al. 2016) flagships, have been proposed to first find Earth-analogs around the closest stars and then to characterize their atmospheres in a search for signs of life.

The extreme planet-star contrast level and the small angular separation for the majority of potential targets present daunting challenges, but our closest solar-like neighbors $\alpha$ Cen A and B (G2 and K1V, respectively) offer some relief to these problems. At $\alpha$ Cen's distance of $1.34 \mathrm{pc}$ the habitable zone $(\sim 1.2 \mathrm{AU}$ for $\alpha$ Cen A) has an angular separation of $0.9^{\prime \prime}$. The contrast ratio problem is greatly reduced by operation in the mid-infrared where a $260 \mathrm{~K}, 1 \mathrm{R}_{\oplus}$ Earth-twin emits $\sim 20 \mu \mathrm{Jy}$. As a free-floating object such an Earth-twin would be easily detectable in a hour with MIRI imaging at $11.3 \mu \mathrm{m}$. The problem, of course, is the presence of not just one star, $\alpha$ Cen A, which can be blocked by the FQPM coronagraph, but the companion $\alpha$ Cen B which during the early years of JWST's mission sits only $\sim 5^{\prime \prime}$ away (Kervella et al.2016) and cannot be fully blocked.

With a modest investment in observing time, perhaps 100 hours, and with a great deal of post-processing MIRI could detect a $1 \mathrm{R}_{\oplus}$ planet in the $\mathrm{HZ}$ of either star. The left-hand panel of Figure 11 shows the flux density for an Earth-twin as a function of separation from $\alpha$ Cen A. The solid curves in the right-hand panel show the signal-to-noise-ratio (SNR) as a function of separation and incorporate the usual noise terms (zodiacal background, read noise, shot noise from the star and planet as 
attenuated by the coronagraph, and residual speckle structure). The different colors represent increasing levels of speckle suppression via post-processing. The dotted curves convey the same information but with the addition of noise terms due to the unattenuated presence of $\alpha$ Cen B located on the opposite side of $\alpha$ Cen A from the putative planet. Ignored in this analysis are various detector pathologies and the detailed spatial structure of the PSF.

Whether the required level of speckle suppression can be achieved, especially in the presence of $\alpha$ Cen B, is open to question. However, the enormous sensitivity of JWST at thermal IR wavelengths provides an significant advantage relative to ground-based observatories such as the VLT which is presently planning a "Breakthrough" program to search for Earths orbiting $\alpha$ Cen A or B (ESO|2016).
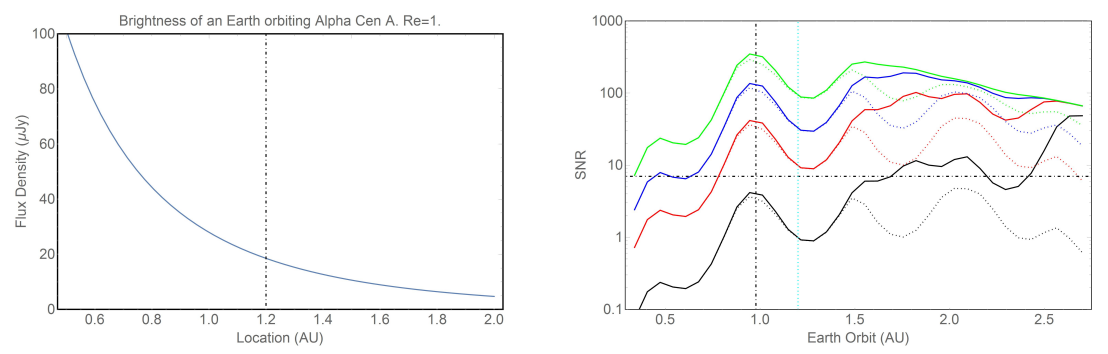

Fig. 11 Left) The flux density for an Earth-twin orbiting $\alpha$ Cen A as a function of separation. The vertical line denotes the center of the HZ. Right) The SNR for an Earth-twin after 25 hours of integration under a number of assumptions. The solid curves ignore the influence $\alpha$ Cen $\mathrm{B}$ and show the SNR for an Earth-twin as a function of separation for different levels of speckle subtraction: from bottom to top, black (nominal Boccaletti et al. (2015) and $10 \times($ red), $30 \times$ (blue) and $100 \times$ (green) improvements in suppression relative to the nominal. The dotted lines show what happens when the influence $\alpha$ Cen B located $5^{\prime \prime}$ away (on the opposite side from the planet) is included. The vertical lines denote the nominal Inner Working Angle of $2 \lambda / \mathrm{D}$ (black) and the location of the center of the Habitable Zone (cyan).

\section{Characterizing the Atmosphere of the Nearest Transiting Habitable Earth}

As noted above, $\mathrm{M}$ dwarf hosts will have the most favorable Earth-like planets for atmospheric characterization via transit techniques. Characterization via transmission spectroscopy will be exceptionally difficult because of the minuscule signals ( $\sim 10$ ppm or less; see above). Emission measurements may be more promising. The TRAPPIST-1 d planet has radius $0.772 R_{\oplus}$ and equilibrium temperature $288 \mathrm{~K}$ (Gillon et al.2017). If a blackbody, its continuum emission would be $20-60 \mathrm{ppm}$ compared to its star at wavelengths $10-15 \mu \mathrm{m}$. This is the optimal wavelength 
range for JWST emission observations; the planet's flux is too low at shorter wavelengths, and the telescope + zodiacal background is too high at longer wavelengths (Glasse et al.2015). JWST MIRI would detect this emission at $\mathrm{S} / \mathrm{N}>3$ in $50 \mathrm{sec}-$ ondary eclipses ( $49 \mathrm{~m}$ each plus equal time on the star + planet) in its $12.8 \mu \mathrm{m}$ and $15 \mu \mathrm{m}$ filters. A detection at $12.8 \mu \mathrm{m}$ and a non-detection at $15 \mu \mathrm{m}$ would indicate a strong Earth-like $\mathrm{CO}_{2}$ feature in the planet's atmosphere. These observations could be accomplished in under 500 hours total, only about $5 \%$ of the time in 1 year. Discovery of a closer ultracool dwarf system with transiting planets would reduce this time further.

The newly-discovered super-Earth LHS 1140 b (Dittmann et al. 2017) would require a comparable amount of total time for similar MIRI measurements. Its cooler temperature $T_{\mathrm{eq}}=230 \mathrm{~K}$ and higher luminosity host star are nearly balanced by its longer transit duration and larger radius. However, LHS $1140 \mathrm{~b}$ is not an optimal target for emission observations because the timing of its secondary eclipse is not (yet) known, and the eccentricity of its $24.7 \mathrm{~d}$ orbit is uncertain and potentially large $(e<0.29)$. The TRAPPIST-1 planets all have low eccentricities, so this is not an issue for them.

Acknowledgements We acknowledge valuable discussions with George and Marcia Rieke, Everett Schlawin, Pierre-Oliver Lagage, and Rene Doyon.

Some of the research described in this publication was carried out in part at the Jet Propulsion Laboratory, California Institute of Technology, under a contract with the National Aeronautics and Space Administration. TPG acknowledges support from the JWST project for this work.

\section{References}

Acke B, Min M, Dominik C et al. (2012) Herschel images of Fomalhaut. An extrasolar Kuiper belt at the height of its dynamical activity. A\&A540:A125

Artigau É, Sivaramakrishnan A, Greenbaum AZ et al. (2014) NIRISS aperture masking interferometry: an overview of science opportunities. In: Space Telescopes and Instrumentation 2014: Optical, Infrared, and Millimeter Wave, Proc SPIE, vol 9143, p 914340, DOI 10.1117/12.2055191

Barstow JK Irwin PGJ (2016) Habitable worlds with JWST: transit spectroscopy of the TRAPPIST1 system? MNRAS461:L92-L96

Barstow JK, Aigrain S, Irwin PGJ, Kendrew S Fletcher LN (2015) Transit spectroscopy with James Webb Space Telescope: systematics, starspots and stitching. MNRAS448:2546-2561

Barstow JK, Aigrain S, Irwin PGJ, Kendrew S Fletcher LN (2016) Telling twins apart: exo-Earths and Venuses with transit spectroscopy. MNRAS458:2657-2666

Batalha NE Line MR (2017) Information Content Analysis for Selection of Optimal JWST Observing Modes for Transiting Exoplanet Atmospheres. AJ153:151

Beichman C, Benneke B, Knutson H et al. (2014a) Observations of Transiting Exoplanets with the James Webb Space Telescope (JWST). PASP126:1134

Beichman C, Gelino CR, Kirkpatrick JD et al. (2014b) WISE Y Dwarfs as Probes of the Brown Dwarf-Exoplanet Connection. ApJ783:68

Beichman CA, Fridlund M, Traub WA et al. (2007) Comparative Planetology and the Search for Life Beyond the Solar System. Protostars and Planets V pp 915-928

Beichman CA, Krist J, Trauger JT et al. (2010) Imaging Young Giant Planets From Ground and Space. PASP122:162 
Benneke B (2015) Strict Upper Limits on the Carbon-to-Oxygen Ratios of Eight Hot Jupiters from Self-Consistent Atmospheric Retrieval. ArXiv e-prints

Biller BA, Vos J, Bonavita M et al. (2015) Variability in a Young, L/T Transition Planetary-mass Object. ApJ813:L23

Boccaletti A, Lagage PO, Baudoz P et al. (2015) The Mid-Infrared Instrument for the James Webb Space Telescope, V: Predicted Performance of the MIRI Coronagraphs. PASP127:633

Burrows A, Hubbard WB Lunine JI (1989) Theoretical models of very low mass stars and brown dwarfs. ApJ345:939-958

Crepp JR, Rice EL, Veicht A et al. (2015) Direct Spectrum of the Benchmark T Dwarf HD 19467 B. ApJ798:L43

Crossfield IJM (2015) Observations of Exoplanet Atmospheres. PASP127:941

Deming D, Seager S, Winn J et al. (2009) Discovery and Characterization of Transiting Super Earths Using an All-Sky Transit Survey and Follow-up by the James Webb Space Telescope. PASP121:952

Dittmann JA, Irwin JM, Charbonneau D et al. (2017) A temperate rocky super-Earth transiting a nearby cool star. Nature 544:333 - 336

Domagal-Goldman SD, Roberge A, Arney GN et al. (2017) The Next Generation of Observations of Planets Beyond Our Solar System. LPI Contributions 1989:8189

Dong S Zhu Z (2013) Fast Rise of "Neptune-size" Planets (4-8 $\mathrm{R}_{\oplus}$ ) from P 10 to 250 Days Statistics of Kepler Planet Candidates up to 0.75 AU. ApJ778:53

Doyon R, Hutchings JB, Beaulieu M et al. (2012) The JWST Fine Guidance Sensor (FGS) and Near-Infrared Imager and Slitless Spectrograph (NIRISS). In: Space Telescopes and Instrumentation 2012: Optical, Infrared, and Millimeter Wave, Proc SPIE, vol 8442, p 84422R, DOI $10.1117 / 12.926578$

Dressing CD Charbonneau D (2015) The Occurrence of Potentially Habitable Planets Orbiting M Dwarfs Estimated from the Full Kepler Dataset and an Empirical Measurement of the Detection Sensitivity. ApJ807:45

Ertel S, Wolf S Rodmann J (2012) Observing planet-disk interaction in debris disks. A\&A544:A61

ESO (2016) Eso news release. URL https://www.eso.org/public/unitedkingdom/ news/eso1702/

Faherty JK, Burgasser AJ, Walter FM et al. (2012) The Brown Dwarf Kinematics Project (BDKP). III. Parallaxes for 70 Ultracool Dwarfs. ApJ752:56

Ferruit P, Bagnasco G, Barho R et al. (2012) The JWST near-infrared spectrograph NIRSpec: status. In: Space Telescopes and Instrumentation 2012: Optical, Infrared, and Millimeter Wave, Proc SPIE, vol 8442, p 84422O, DOI 10.1117/12.925810

Ferruit P, Birkmann S, Böker T et al. (2014) Observing transiting exoplanets with JWST/NIRSpec. In: Space Telescopes and Instrumentation 2014: Optical, Infrared, and Millimeter Wave, Proc SPIE, vol 9143, p 91430A, DOI 10.1117/12.2054756

Fridlund MC (2004) Darwin and TPF: technology and prospects. In: Traub WA (ed) New Frontiers in Stellar Interferometry, Proc SPIE, vol 5491, p 227, DOI 10.1117/12.550782

Gagné J, Lafrenière D, Doyon R et al. (2014) SIMP J2154-1055: A New Low-gravity L4 $\beta$ Brown Dwarf Candidate Member of the Argus Association. ApJ792:L17

Gardner JP (2009) The James Webb Space Telescope. In: Onaka T, White GJ, Nakagawa T Yamamura I (eds) AKARI, a Light to Illuminate the Misty Universe, Astronomical Society of the Pacific Conference Series, vol 418, p 365

Gillon M, Triaud AHMJ, Demory BO et al. (2017) Seven temperate terrestrial planets around the nearby ultracool dwarf star TRAPPIST-1. Nature542:456-460

Glasse A, Rieke GH, Bauwens E et al. (2015) The Mid-Infrared Instrument for the James Webb Space Telescope, IX: Predicted Sensitivity. PASP127:686

Greene TP, Line MR, Montero C et al. (2016) Characterizing Transiting Exoplanet Atmospheres with JWST. ApJ817:17

Howe AR, Burrows A Deming D (2017) An Information-theoretic Approach to Optimize JWST Observations and Retrievals of Transiting Exoplanet Atmospheres. ApJ835:96 
Janson M, Quanz SP, Carson JC et al. (2015) High-contrast imaging with Spitzer: deep observations of Vega, Fomalhaut, and $\varepsilon$ Eridani. A\&A574:A120

Kalas P, Graham JR, Chiang E et al. (2008) Optical Images of an Exosolar Planet 25 Light-Years from Earth. Science 322:1345

Kendrew S, Scheithauer S, Bouchet P et al. (2015) The Mid-Infrared Instrument for the James Webb Space Telescope, IV: The Low-Resolution Spectrometer. PASP127:623

Kervella P, Mignard F, Mérand A Thévenin F (2016) Close stellar conjunctions of $\alpha$ Centauri A and B until 2050. An $\mathrm{m}_{K}=7.8$ star may enter the Einstein ring of $\alpha$ Cen A in 2028. A\&A594:A107

Kirkpatrick JD (2013) Cold brown dwarfs with WISE: Y dwarfs and the field mass function. Astronomische Nachrichten 334:26-31

Kirkpatrick JD, Reid IN, Liebert J et al. (1999) Dwarfs Cooler than "M": The Definition of Spectral Type "L" Using Discoveries from the 2 Micron All-Sky Survey (2MASS). ApJ519:802-833

Kirkpatrick JD, Cushing MC, Gelino CR et al. (2011) The First Hundred Brown Dwarfs Discovered by the Wide-field Infrared Survey Explorer (WISE). ApJS197:19

Konopacky QM, Barman TS, Macintosh BA Marois C (2013) Detection of Carbon Monoxide and Water Absorption Lines in an Exoplanet Atmosphere. Science 339:1398-1401

Kreidberg L Loeb A (2016) Prospects for Characterizing the Atmosphere of Proxima Centauri b. ApJ832:L12

Kreidberg L, Bean JL, Désert JM et al. (2014) A Precise Water Abundance Measurement for the Hot Jupiter WASP-43b. ApJ793:L27

Krist JE, Balasubramanian K, Muller RE et al. (2010) The JWST/NIRCam coronagraph flight occulters. In: Space Telescopes and Instrumentation 2010: Optical, Infrared, and Millimeter Wave, Proc SPIE, vol 7731, p 77313J, DOI 10.1117/12.856488

Krist JE, Stapelfeldt KR, Bryden G Plavchan P (2012) Hubble Space Telescope Observations of the HD 202628 Debris Disk. AJ144:45

Lagrange AM, Bonnefoy M, Chauvin G et al. (2010) A Giant Planet Imaged in the Disk of the Young Star $\beta$ Pictoris. Science 329:57

Lawler SM, Greenstreet S Gladman B (2015) Fomalhaut b as a Dust Cloud: Frequent Collisions within the Fomalhaut Disk. ApJ802:L20

Line MR Yung YL (2013) A Systematic Retrieval Analysis of Secondary Eclipse Spectra. III. Diagnosing Chemical Disequilibrium in Planetary Atmospheres. ApJ779:3

Line MR, Wolf AS, Zhang X et al. (2013) A Systematic Retrieval Analysis of Secondary Eclipse Spectra. I. A Comparison of Atmospheric Retrieval Techniques. The Astrophysical Journal 775:137

Line MR, Knutson H, Wolf AS Yung YL (2014) A Systematic Retrieval Analysis of Secondary Eclipse Spectra. II. A Uniform Analysis of Nine Planets and their C to O Ratios. The Astrophysical Journal 783:70

Lisse CM, Wyatt MC, Chen CH et al. (2012) Spitzer Evidence for a Late-heavy Bombardment and the Formation of Ureilites in $\eta$ Corvi at $\sim 1$ Gyr. ApJ 747:93

Luhman KL (2014) Discovery of a $\sim 250$ K Brown Dwarf at 2 pc from the Sun. ApJ786:L18

Luhman KL, Adame L, D'Alessio P et al. (2005) Discovery of a Planetary-Mass Brown Dwarf with a Circumstellar Disk. ApJ635:L93-L96

Luhman KL, Burgasser AJ, Labbé I et al. (2012) Confirmation of One of the Coldest Known Brown Dwarfs. ApJ744:135

Marley MS, Fortney JJ, Hubickyj O, Bodenheimer P Lissauer JJ (2007) On the Luminosity of Young Jupiters. ApJ655:541-549

Mayor M Queloz D (1995) A Jupiter-mass companion to a solar-type star. Nature378:355-359

Mennesson B, Gaudi S, Seager S et al. (2016) The Habitable Exoplanet (HabEx) Imaging Mission: preliminary science drivers and technical requirements. In: Space Telescopes and Instrumentation 2016: Optical, Infrared, and Millimeter Wave, Proc SPIE, vol 9904, p 99040L, DOI $10.1117 / 12.2240457$

Mollière P, van Boekel R, Bouwman J et al. (2017) Observing transiting planets with JWST. Prime targets and their synthetic spectral observations. A\&A600:A10 
Mordasini C, van Boekel R, Mollière P, Henning T Benneke B (2016) The Imprint of Exoplanet Formation History on Observable Present-day Spectra of Hot Jupiters. ApJ832:41

Nakajima T, Oppenheimer BR, Kulkarni SR et al. (1995) Discovery of a cool brown dwarf. Nature378:463-465

Öberg KI, Murray-Clay R Bergin EA (2011) The Effects of Snowlines on C/O in Planetary Atmospheres. ApJ743:L16

Patel RI, Metchev SA Heinze A (2014) A Sensitive Identification of Warm Debris Disks in the Solar Neighborhood through Precise Calibration of Saturated WISE Photometry. ApJS212:10

Rieke GH, Wright GS, Böker T et al. (2015) The Mid-Infrared Instrument for the James Webb Space Telescope, I: Introduction. PASP127:584

Rieke MJ, Kelly D Horner S (2005) Overview of James Webb Space Telescope and NIRCam's Role. In: Heaney JB Burriesci LG (eds) Cryogenic Optical Systems and Instruments XI, Proc SPIE, vol 5904, pp 1-8, DOI 10.1117/12.615554

Rocchetto M, Waldmann IP, Venot O, Lagage PO Tinetti G (2016) Exploring Biases of Atmospheric Retrievals in Simulated JWST Transmission Spectra of Hot Jupiters. ApJ833:120

Sallum S, Follette KB, Eisner JA et al. (2015) Accreting protoplanets in the LkCa 15 transition disk. Nature 527:342-344

Schlawin E, Rieke M, Leisenring J et al. (2017) Two NIRCam Channels are Better than One: How JWST Can Do More Science with NIRCam's Short-wavelength Dispersed Hartmann Sensor. PASP129(1):015,001

Schlieder JE, Beichman CA, Meyer MR Greene T (2015) Toward Direct Imaging of Low-Mass Gas-Giants with the James Webb Space Telescope. ArXiv e-prints

Schneider AC, Cushing MC, Kirkpatrick JD et al. (2015) Hubble Space Telescope Spectroscopy of Brown Dwarfs Discovered with the Wide-field Infrared Survey Explorer. ApJ804:92

Schwieterman EW, Meadows VS, Domagal-Goldman SD et al. (2016) Identifying Planetary Biosignature Impostors: Spectral Features of $\mathrm{CO}$ and $\mathrm{O}_{4}$ Resulting from Abiotic $\mathrm{O}_{2} / \mathrm{O}_{3}$ Production. ApJ819:L13

Seager S, Turnbull M, Sparks W et al. (2015) The Exo-S probe class starshade mission. In: Techniques and Instrumentation for Detection of Exoplanets VII, Proc SPIE, vol 9605, p 96050W, DOI 10.1117/12.2190378

Shabram M, Fortney JJ, Greene TP Freedman RS (2011) Transmission Spectra of Transiting Planet Atmospheres: Model Validation and Simulations of the Hot Neptune GJ 436b for the James Webb Space Telescope. ApJ727:65

Sing DK, Fortney JJ, Nikolov N et al. (2016) A continuum from clear to cloudy hot-Jupiter exoplanets without primordial water depletion. Nature529:59-62

Spiegel DS Burrows A (2012) Spectral and Photometric Diagnostics of Giant Planet Formation Scenarios. ApJ745:174

Stark CC Kuchner MJ (2008) The Detectability of Exo-Earths and Super-Earths Via Resonant Signatures in Exozodiacal Clouds. ApJ686:637-648

Su K (2015) Probing Terrestrial Planet Formation by Witnessing Large Collisions in Extreme Debris Disks. In: AAS/Division for Extreme Solar Systems Abstracts, AAS/Division for Extreme Solar Systems Abstracts, vol 3, p 120.11

Su KYL, Rieke GH, Malhotra R et al. (2013) Asteroid Belts in Debris Disk Twins: Vega and Fomalhaut. ApJ763:118

Wyatt MC (2006) Dust in Resonant Extrasolar Kuiper Belts: Grain Size and Wavelength Dependence of Disk Structure. ApJ639:1153-1165

Wyatt MC (2008) Evolution of Debris Disks. ARA\&A46:339-383 